\title{
Gorillas (Gorilla gorilla gorilla) Fail to Learn Abstract Cues of Differential Outcomes in a Novel Cognitive Bias Test
}

\author{
Molly C. McGuire and Jennifer Vonk* \\ Oakland University, Department of Psychology, Rochester, MI, USA \\ *Corresponding author (Email: vonk@oakland.edu)
}

Citation - McGuire, M.C., \& Vonk, J. (2018). Gorillas (Gorilla gorilla gorilla) fail to learn abstract cues of differential outcomes in a novel cognitive bias test. Animal Behavior and Cognition, 5(1), $103-117$. https://doi.org/10.26451/abc.05.01.08.2018

\begin{abstract}
In order to assess mood state in three male western lowland gorillas housed in a bachelor group, we developed a novel version of a cognitive bias task. The background color of a touchscreen presented a conditional 'if, then..." rule relating to outcomes involving differential amounts of food rewards. The gorillas struggled to reach a criterion of $80 \%$ responding required for testing. In follow-up experiments, we assessed whether the gorillas did in fact prefer three pieces of food to one piece of food when presented with images of food quantities and actual food quantities. Gorillas did not learn to select the stimulus that indicated three food items over one food item when we used images of actual quantities of the rewarded food but they did prefer three actual pieces of food. Thus, like other primates, gorillas may respond differently to quantities of edible and inedible items. In addition, they may struggle with learning conditional discriminations when cues to differential responses and outcomes are arbitrary rather than intuitively connected. These studies highlight the importance of methodology when devising tests to assess cognition or affect in nonhuman primates.
\end{abstract}

Keywords - Gorillas, Affect, Cognitive bias, Quantity, Conditional discrimination

Cognitive bias in animals (as well as humans) can be described as a bias in cognitive processing due to changes in affective state. More specifically, the tendency to interpret neutral or ambiguous stimuli in a negative manner is considered pessimism, whereas the tendency to interpret neutral or ambiguous stimuli in a positive manner is considered optimism. Humans that display a negative cognitive bias are more likely to develop and maintain mood disorders such as anxiety and depression (Hallion \& Ruscio, 2011). Therefore, cognitive bias tasks may serve an important role in assessing psychological well-being.

In humans, cognitive bias is often assessed using verbal exercises. Participants given ambiguous sentences (e.g., "They discussed the priest's strong convictions") are asked to make various judgements about the meaning of those sentences; for example, to choose between a positive interpretation, "They discussed the priest's strong beliefs" or a negative interpretation, "They discussed the priest's criminal record" (Hallion \& Ruscio, 2011, p. 2). These verbal exercises have been used to assess existing cognitive biases and to manipulate cognitive biases. By manipulating the valence of the primer sentences that participants read, researchers have induced negative or positive cognitive biases in healthy populations, causing the participants to interpret ambiguous statements as either threatening or benign (Hallion \& Ruscio, 2011; Mathews et al., 2000). Cognitive bias tasks such as these have even been developed into a form of cognitive bias modification therapy as a way of treating maladaptive cognitive biases in unhealthy populations (Hallion \& Ruscio, 2011). Clearly, cognitive bias assessments are a useful tool in the measurement and manipulation of human biases. 
Cognitive bias tasks have also been promoted as a valuable method of assessing the psychological well-being of animals (e.g., Harding, Paul, \& Mendl, 2004; Mendl \& Paul, 2004; Paul, Harding, \& Mendl, 2005), and have been applied to a variety of species such as rats (Burman, Parker, Paul, \& Mendl, 2008), starlings (Matheson, Asher, \& Bateson, 2008), pigs (Douglas, Bateson, Walsh, Bédué, \& Edwards, 2012), goats (Briefer \& McElligott, 2013), horses (Briefer-Freymond et al., 2014), dogs (Burman et al., 2011), bears (Keen et al., 2014), rhesus macaques (Bethell, Holmes, Maclarnon, \& Semple, 2012), and chimpanzees (Bateson \& Nettle, 2015). Unlike studies of cognitive bias in humans, studies of cognitive bias in nonhumans must rely on nonverbal exercises, which can make interpretation of the results more challenging. However, there is solid evidence that an animal's reactions to neutral or ambiguous stimuli can be influenced by the animal's internal affective state (e.g., Mendl, Burman, Parker, \& Paul, 2009).

One of the most common cognitive bias assessments for animals makes use of a go/no-go design, in which an animal is trained to give a specific response (approach) when presented with one cue and to refrain from responding (do not approach) when presented with a second cue. After animals learn to discriminate between the go and no-go cues, they undergo some sort of condition or treatment meant to induce a specific affective state and are then presented with probe trials in which the stimulus is ambiguous relative to the trained cues. The ambiguous stimuli typically occur in an intermediate spatial location, or are intermediate along a perceptual continuum. For example, in Burman et al. (2011), dogs learned to discriminate between black and white food bowls. One bowl always contained food and was the "go" choice, the other never contained food and was the "no-go" choice. The dogs then experienced either a positive condition meant to elicit positive affect or a neutral condition as a control. Various grey bowls were introduced in testing. If the dogs treated the grey bowls as reward bowls by approaching quickly, they were considered optimistic, whereas if they treated them as non-rewarded (i.e., approached slowly or not at all) they were considered pessimistic. By comparing responses to the ambiguous probe(s) to responses to the trained cues, researchers can gain insight into cognitive biases. That is, if animals treat the ambiguous probe cue as they do the "go" stimulus, they are inferred to be displaying optimism or positive cognitive bias; whereas, if they respond to the probe as they do to the "no-go" stimulus they are inferred to be displaying pessimism or a negative cognitive bias.

It has been suggested that go/no-go tasks are susceptible to a reduction in response rates, not due to changes in mood but due to the fact that the animals have learned when rewards are and are not available. As a consequence, at times they choose to "play it safe" and conserve energy instead of responding (Mendl et al., 2009). One way to address this challenge is to require responding to both stimuli but to reward responses to only one of those stimuli as in Bateson and Nettle (2015). Another option is to present a choice between two stimuli and to reward responses to one and not the other - a strategy also adopted by Bateson and Nettle (2015). Most relevant to the current study, Keen et al. (2014) examined the effects of enrichment on cognitive bias in grizzly bears by requiring active responding to two stimuli. These researchers provided positive reinforcement for both responses; the difference being the quantity of food awarded for a correct response to each stimulus. In this manner, the cue signaling the greater amount of food, and its associated response, should be indicative of optimism. Although the bears in this study did not show the expected optimism following enrichment, this method seems promising for use with other species.

Although cognitive bias paradigms have been presented to a variety of species ranging from honeybees (Anderson, Hardcastle, Munafò, \& Robinson., 2012) to monkeys (Bethell et al., 2012; Pomerantz, Terkel, Suomi, \& Paukner, 2012), only a handful of studies have investigated cognitive bias in apes; in chimpanzees (Allritz, Call, \& Borkenau, 2016; Bateson \& Nettle, 2015) and our own previous work with gorillas (McGuire, Vonk, Fuller, \& Allard, 2017a; McGuire, Vonk, \& Johnson-Ulrich, 2017b). We were interested in assessing emotional states in a group of bachelor silverback gorillas because they comprise a relatively young group of silverback gorillas that spend a fair bit of time engaging in agonistic behavior, as they attempt to shift or reaffirm their position in the dominance hierarchy. They also exhibit some variance in their indoor and outdoor habitats across the seasons, and we were interested in how such variables might affect their mood states, and indeed, whether they would demonstrate stable or fluctuating mood states across our tests. 
We chose to adopt a methodology similar to that used by Keen et al. (2014) because it seemed relatively easy to implement given that the gorillas had already participated in, but failed to adequately learn, an ambiguous cue paradigm presented on the computer touchscreen (McGuire et al. 2017a; 2017b). They had also previously demonstrated their ability to estimate quantities (Vonk et al., 2014), making it feasible to use differing amounts of food reward as a means to assess optimism. The present experiment utilized a 1:3 ratio of food items based on the training ratios used in Vonk et al. (2014), and Keen et al. (2014) who found that bears responded to food ratios of 1:3, favoring three items over one item.

Similar to Keen et al. (2014), in this study, gorillas were trained on a conditional discrimination in which, when presented with a pair of identical stimuli presented on a black background they were required to respond one way (i.e., touch the stimulus on the right), and when presented with the same two stimuli on a white background, they were required to respond another way (i.e., touch the stimulus on the left). As with Keen et al.'s experiment, our test also made use of differential reinforcements, with correct responses to the white background resulting in three food items and correct responses to the black background resulting in only one. After reaching criterion, the gorillas were to proceed to the testing phase, which would have introduced ambiguous probe trials involving various shades of grey on the background screen meant to assess their cognitive biases. During the testing phase, it was expected that the gorillas would respond more often and more quickly to the left option (the option associated with more food) if they were optimistic when faced with the ambiguous trials.

\section{Experiment 1: Cognitive Bias Training}

\section{Methods}

Subjects. Three adult male western lowland gorillas (Chip, 19 years old; Pende, 18 years old; and Kongo, 17 years old), all half-siblings, participated in this study. These gorillas, housed at the Detroit Zoo (an institution accredited by the Association of Zoos and Aquariums), lived together in an all-male bachelor group. These gorillas had participated in a study where they selected arrays of differing quantities of dots using a touchscreen computer (Vonk et al., 2014). They were also being tested on a variety of preference assessment tasks using the touchscreen concurrently (unpublished data). The research took place three mornings each week when they were temporarily housed individually for their morning meal in an indoor area not accessible to zoo visitors. Training and testing with these animals was approved by the IACUC of Oakland University, and the activities were presented as a form of cognitive enrichment.

Materials. A durable Panasonic Toughbook Laptop CF19 computer and $48.26 \mathrm{~cm}$ VarTech Armorall capacitive touchscreen monitor welded inside a rolling LCD panel cart encased with top and sides comprised the experimental apparatus. Using a $1.2 \mathrm{~m}$ by $1.2 \mathrm{~m}$ plywood ramp, the researchers ensured that the apparatus was positioned so that it was flush with the mesh of the gorilla indoor habitat. When rewarding the gorillas for a correct choice, food items were delivered to the gorilla using a PVC chute attached to the apparatus.

For this task, special high fiber browse biscuits were used as the rewards in an attempt to motivate the gorillas to perform. How much of the reward each gorilla received depended on their performance - any leftover biscuits at the end of the training period were returned to the kitchen. Additionally, when the gorillas' training had concluded (including this task as well as a conditional discrimination task the gorillas were learning at the same time), any leftover food from their breakfast tray was withheld until just before the gorillas were released into the outdoor habitats for the day instead of being fed out at the conclusion of each gorilla's session. It was predicted that withholding the remaining food from their breakfast tray would help to further motivate the gorillas to perform with greater accuracy as, if they performed poorly, they would have to wait a longer time to receive their breakfast. In other words, the better their performance, the more food they would receive right away. By providing a "jackpot" of unused food directly following training, we may have unintentionally reinforced poor performance in prior experiments (e.g., McGuire et al., 2017a). 
Procedure. Gorillas were trained on a cognitive bias task based on quantity differences. The task was a two choice discrimination task programmed in Inquisit Version 3 and presented on a Panasonic Toughbook CF19 projected to a capacitive touchscreen. The background was either black or white and contained two identical pale blue squares vertically aligned on the left or right sides of the screen (Figure 1). When the background was white, the gorilla was rewarded with three pieces of chow for touching the left square. When the background was black, the gorilla was rewarded with one piece of chow for touching the right square. Touches to the incorrect side resulted in no reward. Thus, the task was, in essence, a form of conditional discrimination, which has not been previously assessed in gorillas, although unpublished work in our lab suggests that it may be a difficult task for them to learn. The advantage of this method is that it allowed the gorillas to discriminate choices based on location rather than identity, which is something we expected them to attend to given their spatial learning abilities (Gibeault, \& MacDonald, 2000; MacDonald, 1994). Furthermore, Keen et al. (2014) implemented the basic task successfully with grizzly bears, as noted above.

Training sessions consisted of five white and five black background trials presented in random order. Once the gorillas reached criterion ( $80 \%$ correct for four consecutive sessions) they would have begun the testing phase of the experiment. The gorillas received four training sessions per day, three days a week. We applied this relatively stringent criterion to ensure consistent responding across test days because performance of non-laboratory animals, in particular, can be erratic and we needed to be certain that the contingences had been learned to render the test trials meaningful.

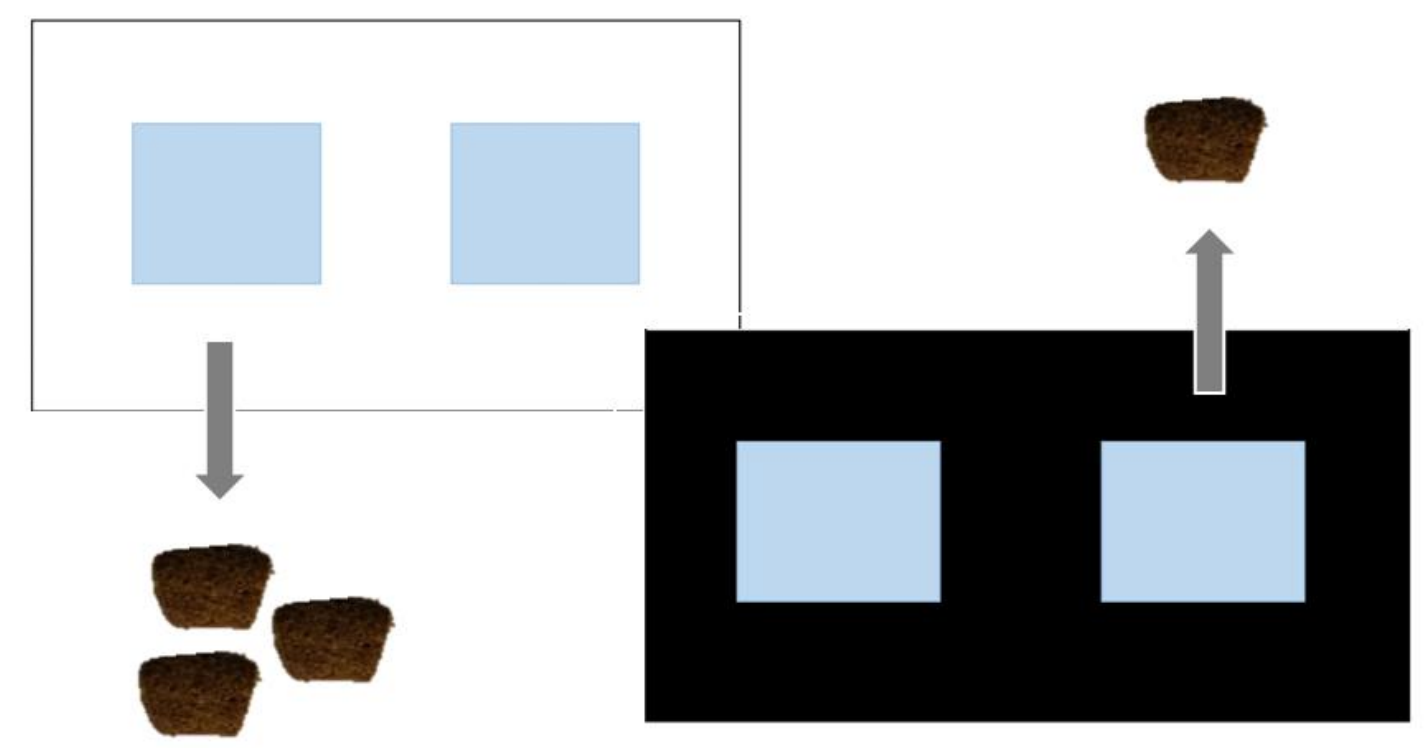

Figure 1. Example training trials; white background trial (top; yielding three pieces of chow), black background trial (bottom; yielding one piece of chow).

\section{Results}

Training was suspended after approximately 300 sessions (or 3000 trials) of this task, in which none of the three gorillas met the criterion for testing. We predicted that, if the gorillas had successfully learned to discriminate between the two conditions, they would have touched the side corresponding to more food rewards (left) faster than they selected the side associated with fewer rewards (right). We assumed that any learning would be most evident later in the training phase. Thus, the following analysis focused on the performance of the gorillas during the final 100 sessions. After eliminating any trials for which the latency exceeded one standard deviation (white: $M=5,811.95 \mathrm{~ms}, S D=23,970.68 \mathrm{~ms}$, black: $M=7,258.49 \mathrm{~ms}, S D=15,681.99 \mathrm{~ms}$ ) above the mean, a paired-samples t-test was conducted to compare latency to respond across background colors (black and white). Taken together, there was not a significant 
difference in latency to touch a square when there was a black background $(M=3,546.77 \mathrm{~ms}, S D=$ $774.81 \mathrm{~ms})$ compared to a white background $(M=3,239.12 .26 \mathrm{~ms}, S D=652.62 \mathrm{~ms}) ; t(2)=1.1, p=.386$, 95\% CI [-895.74, 1511.03]). However, a Wilcoxon signed-rank test showed that Kongo touched the screen significantly faster on white background trials compared to black background trials $(Z=-3.273, p$ $=.001$ ). Neither Chip nor Pende displayed a significant difference in latencies across trial types (both $p s>$ .05 ; Figure 2).

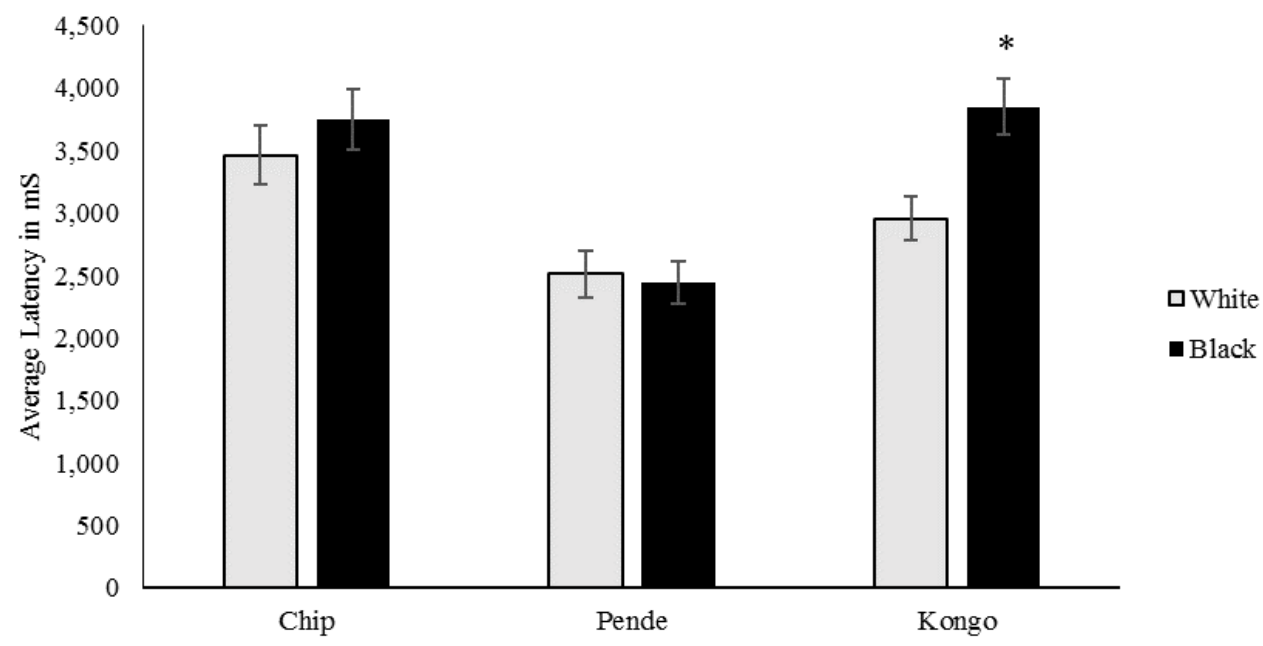

Figure 2. Average latency to touch the screen comparing black versus white background trials for each subject across the last 100 sessions of training $(* p<.05)$.

Binomial testing indicated that, for both black $(N=510, p=.046)$ and white $(N=510, p=.012)$ trials, Kongo performed above chance $(50 \%$ correct). Chip also selected the correct stimulus at a rate above chance on white trials $(N=500, p<.001)$ but at a rate significantly below chance on black trials $(\mathrm{N}=$ $500, p<.001)$. Lastly, binomial tests indicated that Pende selected the correct stimulus at a rate below chance on the white trials $(N=500, p<.001)$ but above chance on the black trials $(N=500, p<.001$; Figure 3).

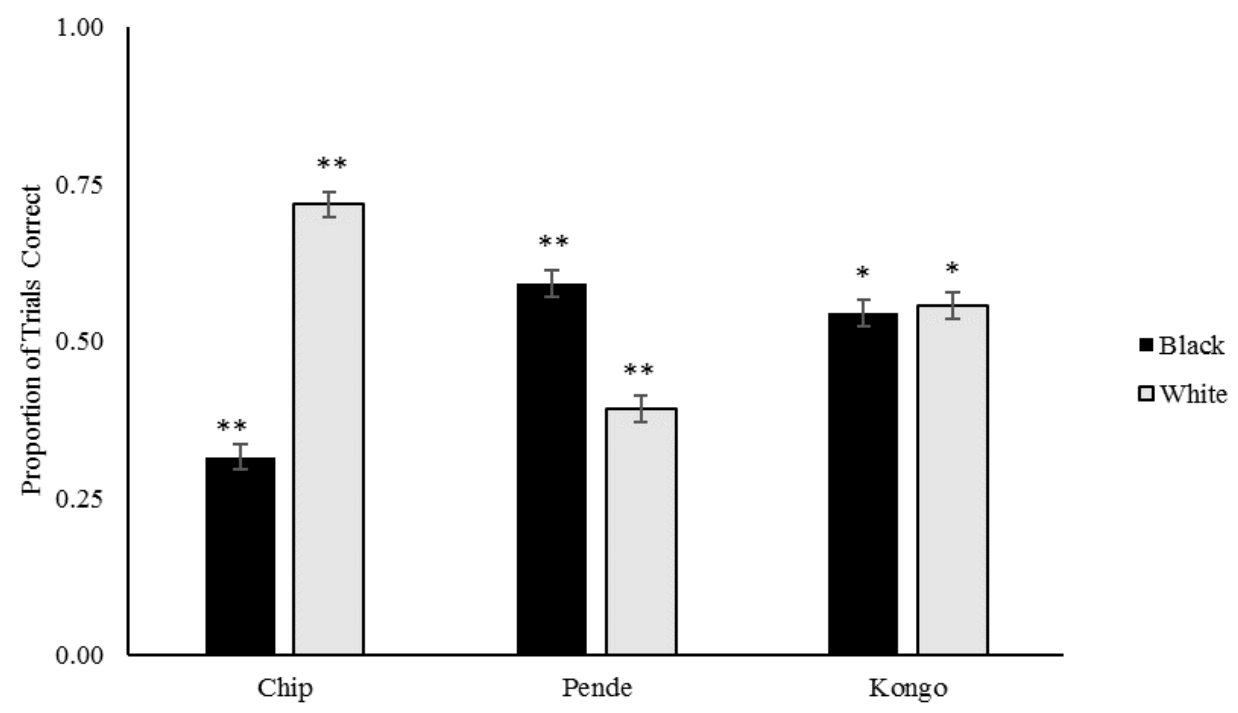

Figure 3. Proportion of correct trials for the last 100 sessions across background colors for each gorilla $\left({ }^{*} p<.05,{ }^{* *} p<.001\right)$. 
These results might be due to the fact that a) gorillas exhibited side biases, b) gorillas might have developed a preference for one stimulus over the other due to the fact that the locations were associated with different quantities of rewards (left side with three pieces and right side with one piece). Indeed, binomial testing indicated that Pende selected the right stimulus $(N=1,000, p<.001)$, and Chip selected the left stimulus $(N=1,000, p<.001)$ at above chance rates. However, Kongo, who was the only gorilla to show above chance levels of accuracy on both types of trial, selected neither the right nor left stimulus at a rate above chance $(50 \% ; N=1,020, p=.731)$. Instead, as indicated in Figure 4 , he may have alternated between preferring to touch the left and right stimuli.
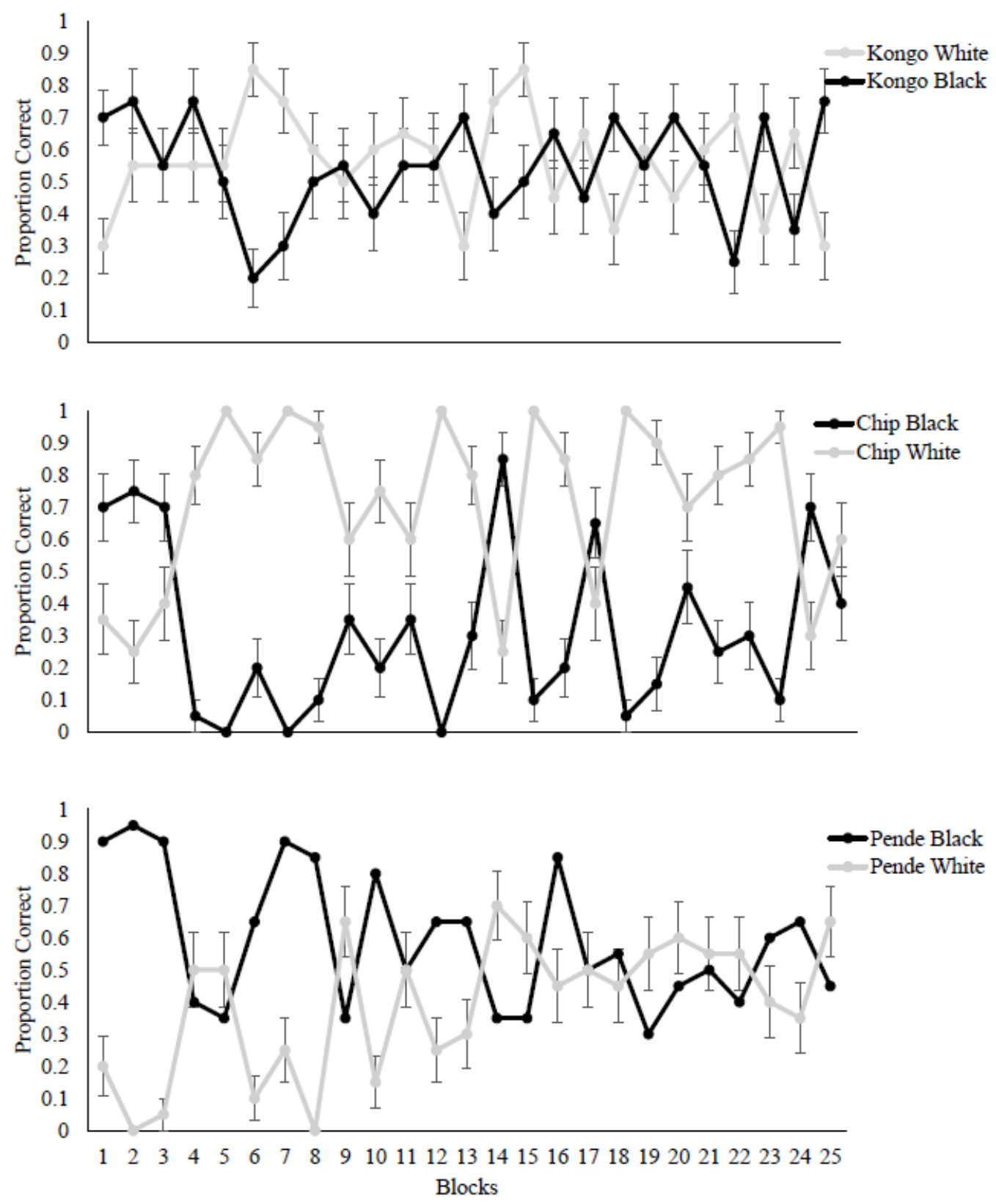

Figure 4. Proportion of correct selections (with SEM) for both black and white background trials during the training phase in blocks of four sessions for each subject. 


\section{Discussion}

These results show that, even though the gorillas failed to reach criterion, Kongo successfully learned the conditional discrimination to some degree, as demonstrated by his reaction times (touching the screen faster on trials with the white background - the background associated with more food) and the fact that he performed above chance on both trial types. Although Kongo's performance was above chance, he did not respond at above chance levels consistently across training sessions, often displaying alternating side biases. Thus, we decided not to present him with testing sessions. Later attempts to retrain him were also unsuccessful.

Chip and Pende, on the other hand, appeared to have developed opposing preferences or side biases. This is evident in the fact that Chip responded above chance on the white trials but significantly below chance on the black trials, possibly indicating a bias for the left stimulus. It is possible that this preference may have been due to the larger food reward associated with the left stimulus, indicating that he did learn that there was a difference in the rewards for left and right stimuli even if he was not able to map this knowledge onto the corresponding background color. The reverse was true of Pende, who performed above chance on the black trials and below chance on the white trials (indicating a bias for the right side option). Pende's bias for the right stimulus may have been due to a preference for less items at a single time, a preference that has been observed in other species including cats, at least with certain ratios of food items (Bánszegi, Urrutia, Szenczi, \& Hudson, 2016). He also responded the most quickly of all of the gorillas. It is possible that he may have been overly eager to receive food rewards and thus never registered that the outcomes differed in quantity.

Conditional discrimination is a complex form of learning. However, many species have been shown capable of conditional discrimination learning including rats (Brown, Pagani, \& Stanton, 2005), octopuses and cuttlefishes (Hvorecny et al., 2007), and chimpanzees (Dugdale \& Lowe, 2000). Thomas and Kerr (1976) used the same colors (black and white) to successfully train squirrel monkeys on a conditional discrimination task in which the monkeys were rewarded for selecting odd objects when presented with a white tray and were rewarded for selecting non-odd objects when presented with a black tray. Hvorency et al. (2007) stated that conditional discrimination abilities may be important for species that require complex navigation skills as they must be able to discriminate between environmental cues while also being able to react to specific environmental contexts (e.g., respond in one way to an environmental landmark under specific circumstances - approach a watering hole when no predators are present - and a different way when encountering the same landmark under different circumstances - do not approach a watering hole when predators are present). However, testing conditional discrimination abilities can be difficult due to the fact that there are many factors that may affect an animal's ability to learn the conditional discrimination such as stimulus salience, the stimulus modality, or the way in which cues are presented (Holland, 1989). For instance, an animal's ability to learn the discrimination may depend on whether the cues are presented simultaneously or serially. It is possible that differential reward amounts, or the background color of the screen, were not salient to the gorillas. In a second experiment we investigated the hypothesis that differential amounts of food may not have been motivating to these gorillas, and we assessed whether they could make the discrimination if more explicit representations of different quantities of food were presented.

\section{Experiment 2: Quantity Preference Assessment with Images}

Only one of the gorillas performed above chance in discriminating between a stimulus that predicted three versus one piece of food reward, and none of the gorillas reached our criterion for consistent accurate responding. In order to best understand the failure of the gorillas to learn this discrimination, we aimed to establish whether they had a preference for three food items compared to one food item. In the absence of this preference, they may not have found the conditions salient. Thus, we assessed whether they could learn the same contingencies if two different stimuli directly representing the same different quantities of food that were being offered were presented via touchscreen. This was done 
in order to clarify whether the gorillas struggled with the concept of differential outcomes associated with different stimuli even if the connection between the outcome and the stimuli was made more explicit. It should be noted that, in order to receive any food at all, the gorillas had to choose the stimulus on the side of the screen that was associated with the background color, so differing preferences for one versus three pieces of food were likely not sufficient to explain the poor performance.

Keen et al. (2014) performed a quantity preference assessment before completing their version of the task with grizzly bears and showed that the bears preferred three apple slices over one apple slice. We assumed that the gorillas would show a similar preference for a higher quantity of food rewards, especially because they had already participated in an experiment establishing that they were able to differentiate between a 3:1 ratio of dot stimuli. In this task, only the gorillas assigned the "choose more" option as correct were capable of learning the task (Vonk et al., 2014) indicating that "more" was the preferred option even without differential food reward outcomes. Additionally, a variety of species such as dogs (Ward \& Smuts, 2007), cats (Bánszegi et al., 2016), and chimpanzees (Beran, McIntyre, Garland, \& Evans, 2013), are capable of discriminating quantities with real food items; therefore, it seemed likely that this capability was also present in gorillas. However, given the failure to perform the conditional discrimination presented in Experiment 1, we decided to conduct a post-hoc assessment of the gorillas' ability to learn to associate a picture of three pieces of chow with three pieces of reward and an image of one piece of chow with one piece of chow as a reward.

\section{Methods}

Subjects. The same gorillas participated in this study.

Materials. The testing apparatus in this study was the same as the one described in Experiment 1. For each test, images were presented in a two alternative forced choice task. Two different discriminations were tested using this task; a "one item versus three items" discrimination and a "zero items versus three items" discrimination. Depending on the version of the task, the two images displayed consisted of either a field occupied by one and a field occupied by three pieces of chow or an empty field ("no food") and a field occupied by three pieces of chow (400 X $600 \mathrm{MP})$. Three different configurations of the chow pieces were used at random during the trials. For Chip and Kongo, selecting either image resulted in the same audio feedback (in this case a pleasant melodic tone) for both tasks. This was done to prevent confusion during the one versus three discrimination when the gorillas received some food no matter what they selected. They received the same number of pieces of chow that were depicted in the image that they selected on each trial. For consistency, we adopted the same strategy of having a positive auditory signal on the zero versus three discrimination. However, Pende struggled to learn this discrimination and the decision was made to try to facilitate his learning by including a distinct auditory tone (buzzer) whenever he chose the "no food" option on the "zero versus three" task beginning with session 58. Food rewards consisted of the same high fiber primate chow that was depicted in the images (see Figure 5).
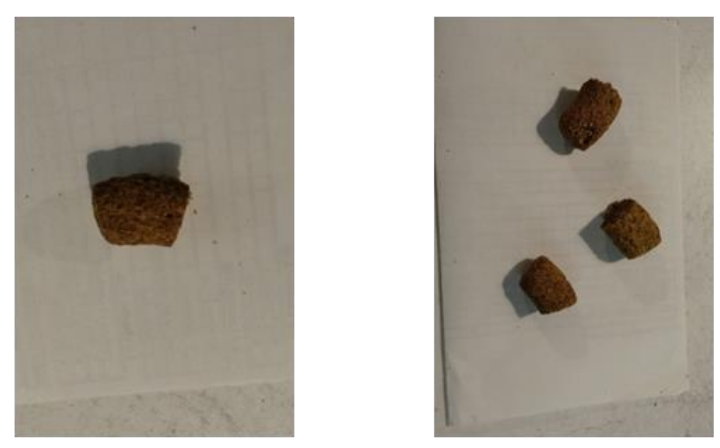

Figure 5. A screen shot of chow images presented to the gorillas in Exp. 2 for the one versus three discrimination. A 'zero' image showed only the background of the paper against the table seen under the chow. 
Procedure. Sessions for all tasks consisted of 20 trials. Each trial presented two alternative images from which the gorillas could select: a "one" image paired with a "three" image or a "zero" image paired with a "three" image. The gorillas were rewarded for touching an image with a corresponding amount of chow. Therefore, any selection of the "zero" image resulted in no food.

Initially, testing began with the one versus three discrimination, because this was the distribution of food rewards that was used in the prior experiment, and the goal was to verify whether the gorillas actually preferred receiving three pieces of chow over one and could use more direct cues (pictures of food) to predict the different outcomes. However, after ten sessions (i.e., 200 trials), it was decided, due to their poor performance, that first it needed to be established that the gorillas would exhibit a preference for "food versus no food." From that point, all three gorillas were presented with the zero versus three discrimination. To return to the "one versus three" task, the gorillas needed to reach criterion of four consecutive sessions of $80 \%$ or better performance, or two consecutive sessions of $90 \%$ or better. If after returning to the "one versus three" discrimination, they failed to reach criterion after 75 trials, rewards were withheld for the "one" choice and a buzzer sound (a sound associated with incorrect responding on previous tasks) was introduced when they selected "one."

\section{Results}

Kongo received seven sessions (140 trials) of "zero versus three" before reaching criterion. Chip received 49 sessions (980 trials) of "zero versus three" before he reached criterion. Pende received 57 sessions $(1,140$ trials) of "zero versus three." Due to poor performance, Pende was then re-introduced to differential feedback (a buzzer sound when selecting the "zero" option) to aid in his learning. He received an additional 44 "buzzer zero versus melodic three" sessions (for a total of 2,010 trials; Figure 6). Although a binomial test indicated that Pende performed above chance on this task across all trials $(N=$ $2,010, p<.001$ ), he did not reach criterion and chose the "three" option only $58 \%$ of the time.

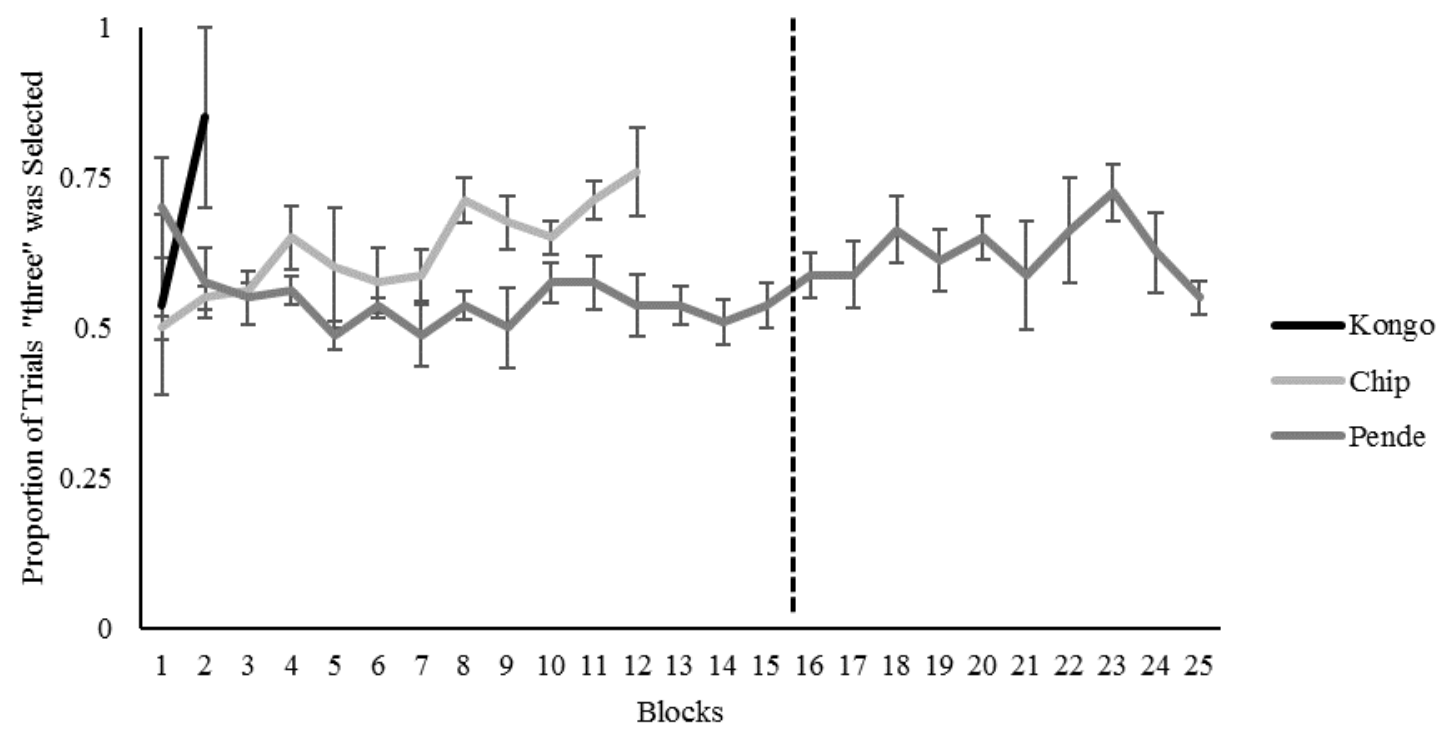

Figure 6. Proportion of trials during the "zero versus three" task that Kongo, Chip and Pende selected the "three" stimulus across blocks of four sessions -The dotted line indicates when an incorrect buzzer was added for "zero" choices for Pende.

After reaching criterion on "zero versus three," Kongo received an additional 110 sessions $(2,200$ trials) of "one versus three." Chip received 48 sessions of "one versus three" followed by 19 sessions of

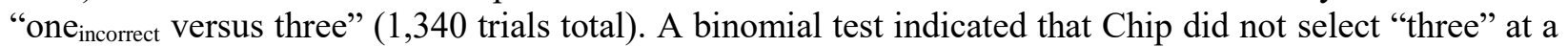
rate above chance across all trials $(N=1,340, p=1.00)$. After 75 trials, Kongo was introduced to the

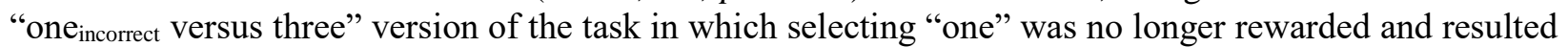

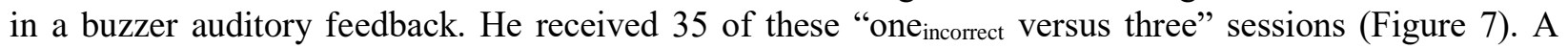


binomial test assessing all trials indicated that he was selecting "three" at a rate above chance $(N=2,200$, $p<.001)$. Although he failed to reach criterion on the "one versus three" task, his performance did appear to improve following implementation of the buzzer feedback and removal of the single chow reward for choices of "one."

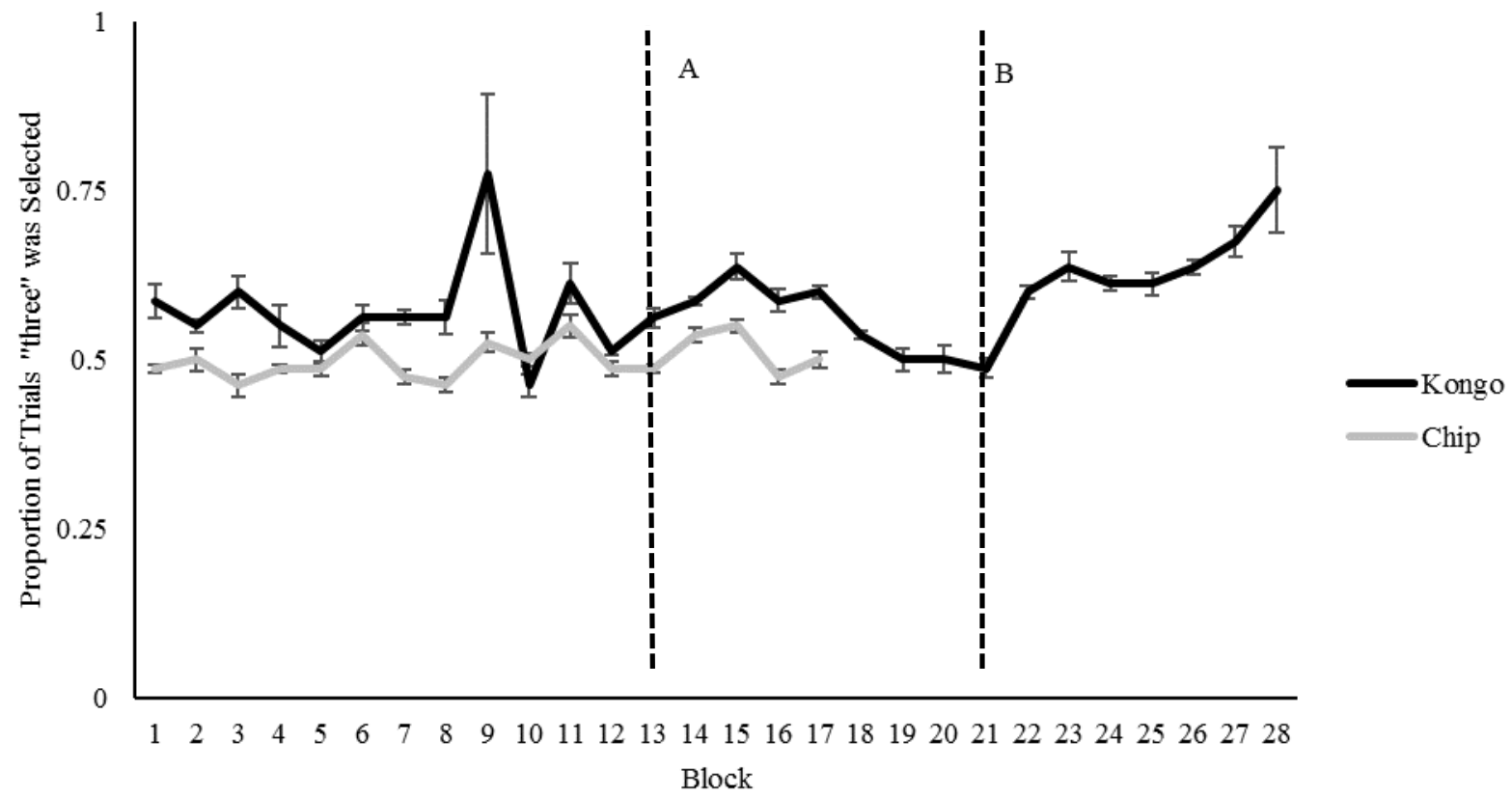

Figure 7. Proportion of trials during the "one versus three" on which Kongo and Chip selected the "three" stimulus across blocks of four sessions- Line A indicates session at which"one incorrect versus three" phase began for Chip, Line B indicates session at

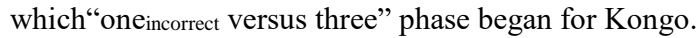

\section{Discussion}

Kongo and Chip reached criterion on the "zero versus three" task, while Pende failed to pass criterion for the "zero versus three" phase of this experiment even after completing over 2,000 trials. He did perform at a rate above chance; however, we terminated testing when we saw a decline rather than continued improvement in his performance. Kongo eventually performed above chance although he did not reach criterion and only showed improvement in the task after we removed a reward of a single chow for touching the corresponding image and introduced the buzzer tone for such choices. Chip did not perform above chance and never reached criterion even after this change.

Interestingly, the gorillas were not biased to spontaneously choose images of three over one piece of chow, although animals, including gorillas, often show a tendency to select more versus fewer dots on a touchscreen (e.g., Vonk et al., 2014). As Pende failed to learn even the "zero versus three" version of this task to criterion, it may be that these gorillas were not very motivated to obtain the type of food reward that was being offered. It is possible that a more valuable reward may have generated the motivation needed to reach criterion on this task. It is surprising that, given the performance of the gorillas on other quantity estimation tasks (Vonk et al., 2014), that they struggled to discriminate between stimuli that both depicted different quantities of food and resulted in differing amounts of actual food being offered. However, previous work has shown that gorillas do not perform as well as orangutans on quantity discrimination tasks (Anderson, Stoinski, Bloomsmith, \& Maple, 2007; Anderson et al., 2005).

Our results are also not the first to present a discrepancy between quantity discrimination performance when choosing between edible and inedible stimuli. For example, Schmitt and Fischer (2011) showed that olive baboons and long-tailed macaques selected higher quantity options more 
frequently when presented with inedible rather than edible items. This difference in responding could not be attributed solely to lack of inhibitory control when presented with edible items, because monkeys did not perform equally poorly when food items presented at choice were replaced with different food reward outcomes. A recent study extended this finding to two New World monkey species - brown capuchin and squirrel monkeys (Gazes, Billas \& Schmitt, 2017). Capuchin monkeys selected the higher quantity more often when presented with edible items but squirrel monkeys performed poorly across all types of stimuli. Increasing the value of the reward improved performance by both species. This study also highlights the fact that different motivations may confound the interpretation of results from different species.

One possible partial explanation for our results would be that the gorillas do not actually prefer three items over one item of food. Bánszegi et al. (2016) investigated the spontaneous food preferences of domestic cats and found that cats did not always choose the larger quantity. They theorized that there may be instinctual motivations influencing their seemingly contradictory responding. For instance, cats may prefer smaller prey items as larger items may be more energetically costly to handle. It may also be that, as domestic cats, they do not typically consume large quantities of food throughout the day and their performance may have reflected this pattern of consumption. Cats may also be motivated to select smaller quantities so as to avoid leaving behind uneaten food that would attract competition from others. It is possible that gorillas prefer smaller portions of food (that they can quickly consume) due to food competition that occurs within the group when they are all housed together in their larger habitats. Gorillas may have adopted the strategy of choosing smaller portions even during testing in light of the food competition that occurs some of the time. However, they did not show a strong preference for the stimulus that predicted the single item either, leaving the mechanism for their performance in question. It seems likely that their performance reflected a failure to learn a conditional discrimination rather than a lack of motivation to receive a greater number of food rewards.

\section{Experiment 3: Quantity Preference Assessment with Food}

In order to test the hypothesis that gorillas might not prefer three over one pieces of chow, we implemented a brief, simple preference test to assess their preference when presented with three versus one actual pieces of chow.

\section{Methods}

Subjects. The same gorillas participated in this study.

Materials. We presented the gorillas with the same type of chow, cut in half, which was presented to the gorillas using two cut PVC tubes with handles that could be used to slide food through the mesh to the gorillas.

Procedure. The experimenter squatted, stood, or sat in front of the mesh separating her from the gorillas. She placed three half pieces of chow in one feeder and one piece of chow in the other feeder and then held the feeders up simultaneously in front of the gorilla placing them equidistant from the gorilla's face to center them. The side presentation of the different quantities was randomized. We presented 23 trials across three days, which was determined by the amount of chow remaining after other tests were conducted. When the gorilla cupped his hand in front of a feeder, it was determined to be a choice and he was given the chow in that feeder. The other feeder was removed and the feeders were replenished for the next trial.

\section{Results}

Binomial tests revealed that Kongo $(M=.78, S D=.42, N=23, p=.01)$ and Pende $(M=.74, S D$ $=.45, N=23, p=.04)$ chose the greater amount of food more often than by chance alone, whereas Chip's tendency to choose the greater amount only approached significance $(M=.70, S D=.47, N=23, p=.09)$. 


\section{Discussion}

The gorillas did show a preference for the larger amount of food when actual food items were presented to them. Kongo, who had the best performance when two-dimensional images of the food amounts were presented, had the strongest preference. Chip, who learned to choose three over zero pieces of chow, did not have a strong preference for three versus one piece of food. Pende who had the weakest performance with the two dimensional stimuli, showed a significant preference for actual food items. These results suggest that a lack of desire for more food items does not completely account for a failure to learn the conditional discrimination in Experiment 1. However, it does appear that the gorillas struggled with the contingency between the images and the differential amounts of food as outcomes, which may have contributed to their difficulty with the task when different spatial locations and background colors signaled differing amounts of food reward. It seems most likely that the use of a conditional discrimination task coupled with a less explicit cue of reward outcomes led to poor performance in the cognitive bias training task.

\section{General Discussion}

In Experiment 1, the gorillas struggled to learn the conditional discrimination, with only one (Kongo) performing statistically above chance on the discrimination and none of them passing the criterion (80\% correct for four consecutive sessions) for continuing to the testing phase of this task. The results of Experiments 2 and 3 offer some insights into the difficulties the gorillas faced while learning the discrimination in Experiment 1. We had assumed, due to the performance of the gorillas on prior quantity estimation tasks indicating that they could discriminate between one and three dot stimuli (Vonk et al., 2014), as well as the findings of Keen et al. (2014) regarding the use of a 1:3 reward ratio on a similar task with grizzly bears, that the food quantity difference offered in this experiment would be salient to the gorillas. Keen et al. (2014) successfully trained grizzly bears on a similar paradigm; rewarding the bears with three apple slices for a high-reward behavior and one slice for a low-reward behavior. The bears in that study required fewer than 600 trials on average to reach $90 \%$ accuracy on the conditional discrimination task (offer the high-reward behavior when presented with cue A and offer the low-reward behavior when presented with cue B). Our assumption may have been misguided in light of the fact that the gorillas did not appear to learn the conditional cue that signaled different correct responses and different outcomes for correct responses. Given that gorillas do show a moderate preference for three pieces over one piece of chow, it is likely that they failed to connect the cues predicting these differential outcomes because they were not explicit enough. Gorillas have also struggled in picture object representation tasks (Parron, Call \& Fagot, 2008), so there may be a conceptual failure when using two-dimensional stimuli to represent real life outcomes. The gorillas had a difficult time distinguishing cues that predicted one and three pieces of chow regardless of whether the cues were background colors or actual images of the amounts of food to be conferred. Thus, their poor performance in Experiment 1cannot solely be attributed to the arbitrary nature of the background color as a cue. Rather, it is possible that gorillas struggle with conditional discriminations when the connection between cues and outcomes is abstract.

Many species have demonstrated the capacity to perform conditional discriminations including other species of great apes. Dugdale and Lowe (2000) successfully trained three chimpanzees on a conditional discrimination task involving the selection of different lexigram icons in order to receive various reinforcing items. In contrast to the chimpanzees in Dugdale and Lowe's (2000) experiment, the gorillas in our study clearly struggled with the conditional discrimination. It is possible that the background of the screen was not viewed as a salient or relevant feature of the task, even though it reliably predicted both different correct response options and different reward outcomes.

It is possible that other external factors may have contributed to their motivation (or lack thereof) to perform these tasks. For instance, the gorillas are frequently adjusting to changes and challenges to the social hierarchy which, at least anecdotally, have been observed to impact their desire to interact with the 
researchers. As adult male gorillas, their primary interest is in establishing dominance and, therefore, it may be that younger or female individuals, or even males in a traditional gorilla group with a harem of females rather than male competitors, would be more motivated to engage in this task than these males were. A young female gorilla also housed in a zoo setting, for example, performed quite well at complex matching-to-sample (Vonk, 2002, 2003, 2013; Vonk \& Hamilton, 2014) and abstract natural categorization tasks (Vonk \& MacDonald, 2002). It is unlikely that there are sex differences in cognition or motivation, rather than situations (such as shifting dominance hierarchies) that influence a gorilla's motivation to respond accurately rather than to merely participate in a cognitive task. Although these male gorillas were willing to participate in testing for approximately 20 minutes each morning (eventually accumulating thousands of trials), they occasionally stopped participating to engage in aggressive displays, periodically fell into patterns of side bias (potentially indicating a lack of attention), and one individual occasionally refused to participate. Anecdotally, we have noticed that these distractions are less likely to occur when the gorillas are presented with tasks that they understand well, as evidenced by consistently high levels of performance. So it may be that the challenge of the task impacts motivation rather than vice versa. Regardless, motivation of subjects to perform accurately on cognitive tasks needs to be considered carefully when interpreting experimental results as indicative of animals' cognitive capacities, especially in settings where animals are not food-adjusted.

Although our active-choice task could not be applied to assess the cognitive biases of the gorillas tested here, we do believe that the task may be usefully implemented with other subjects or species. In fact, we have successfully trained an American black bear to perform the same task (unpublished data), and the bear learned the basic discrimination in fewer than 100 trials. We were then able to present her with test sessions with ambiguous grey backgrounds to assess her optimism. Thus, we are optimistic that these tasks can be successfully applied as a tool to measure cognitive bias.

We believe that the failure of the gorillas in our studies may point to a meaningful difference in the capacity to form representations of real life outcomes from two-dimensional stimuli. The different rate of learning of the one black bear tested and the three gorillas tested here may point to the possibility of species differences in associating images of food and inedible objects to the outcome of receiving real food items. Interestingly, the same bear performed very well on tests of picture-object recognition (Johnson-Ulrich et al., 2016), whereas other gorillas have demonstrated conflicting patterns of results in such studies (Parron, et al., 2008). Whereas the gorillas could direct pointing to actual food items that represented larger quantities, they could not learn to touch images depicting the same quantity differences of the same food items. Their learning was better when greater quantities signaled the presence versus absence of a reward rather than delivery of differential quantities. Thus, our data suggests that gorillas at least those housed in captivity with ready access to food - learn best when there is a direct and salient outcome from a stimulus (receive food, do not receive food) versus an indirect outcome (touch left or touch right in order to receive three or one piece(s) of food). It is possible that gorillas find it more challenging to relate two-dimensional stimuli to the objects they represent, thus failing to associate color cues with different response options or images of food items with differing quantity outcomes. Future studies should assess the representational abilities of gorillas in comparison to other species. Few studies thus far have tested their conceptual abilities with two-dimensional visual stimuli, and most of these previous studies involved a single juvenile gorilla that was very motivated to participate in tasks with human experimenters (Vonk, 2002; 2003; 2013; Vonk \& Hamilton, 2014; Vonk \& MacDonald, 2002). Researchers might consider using real life objects as stimuli and increasing the salience of outcomes in future studies with captive gorillas.

\section{Acknowledgements}

We would like to thank the staff at the Detroit Zoo for facilitating all of the training sessions needed for these experiments and for allowing us to conduct this work. Without the efforts of the animal care staff and Center for Zoo Animal Welfare this work would not have been possible. 


\section{References}

Allritz, M., Call, J., \& Borkenau, P. (2016). How chimpanzees (Pan troglodytes) perform in a modified emotional Stroop task. Animal Cognition, 19, 435-449. http://dx.doi.org/10.1007/s10071-015-0944-3

Anderson, M. H., Hardcastle, C., Munafò, M. R., \& Robinson, E. S. J., (2012). Evaluation of a novel translational task for assessing emotional biases in different species. Cognitive, Affective \& Behavioral Neuroscience 12, 373-381. http://dx.doi.org/10.3758/s13415-011-0076-4

Anderson, U. S., Stoinski, T. S., Bloomsmith, M. A., \& Maple, T. L. (2007). Relative numerousness judgment and summation in young, middle-aged, and older adult orangutans (Pongo pygmaeus abelii and Pongo pygmaeus pygmaeus). Journal of Comparative Psychology, 121, 1-11. http://dx.doi.org/10.1037/0735-7036.121.1.1

Anderson, U. S., Stoinski, T. S., Bloomsmith, M. A., Marr, M. J., Smith, A. D., \& Maple, T. L. (2005). Relative numerousness judgment and summation in young and old Western lowland gorillas. Journal of Comparative Psychology, 119, 285-295. http://dx.doi.org/10.1037/0735-7036.119.3.285

Bánszegi, O., Urrutia, A., Szenczi, P., \& Hudson, R. (2016). More or less: Spontaneous quantity discrimination in the domestic cat. Animal Cognition, 19, 879-888. http://dx.doi.org/10.1007/s10071-016-0985-2

Bateson M., \& Nettle D. (2015) Development of a cognitive bias methodology for measuring low mood in chimpanzees. PeerJ 3:e998. http://dx.doi.org/10.7717/peerj.998

Beran, M. J., McIntyre, J. M., Garland, A., \& Evans, T. A. (2013). What counts for "counting"? Chimpanzees, Pan troglodytes, respond appropriately to relevant and irrelevant information in a quantity judgment task. Animal Behaviour, 85, 987-993. http://dx.doi.org/10.1016/j.anbehav.2013.02.022

Bethell, E., Holmes, A., Maclarnon, A., \& Semple, S. (2012). Cognitive bias in a non-human primate: Husbandry procedures influence cognitive indicators of psychological well-being in captive rhesus macaques. Animal Welfare, 21, 185-195. http://dx.doi.org/10.7120/09627286.21.2.185

Briefer, E. F., \& McElligott, A. G. (2013). Rescued goats at a sanctuary display positive mood after former neglect. Applied Animal Behaviour Science, 146, 45-55. http://dx.doi.org/10.1016/j.applanim.2013.03.007

Briefer Freymond, S., Briefer, E. F., Zollinger, A., Gindrat-von Allmen, Y., Wyss, C., \& Bachmann, I. (2014). Behaviour of horses in a judgment bias test associated with positive or negative reinforcement. Applied Animal Behaviour Science, 158, 34-45. http://dx.doi.org/10.1016/j.applanim.2014.06.006

Brown, K. L., Pagani, J. H., \& Stanton, M. E. (2005). Spatial conditional discrimination learning in developing rats. Developmental Psychobiology, 46, 97-110. http://dx.doi.org/10.1002/dev.20044

Burman, O., McGowan, R., Mendl, M., Norling, Y., Paul, E., ...Keeling, L. (2011). Using judgement bias to measure positive affective state in dogs. Applied Animal Behaviour Science, 132, $160-168$. http://dx.doi.org/10.1016/j.applanim.2011.04.001

Burman, O. H. P., Parker, R., Paul, E. S., \& Mendl, M. (2008). A spatial judgement task to determine background emotional state in laboratory rats, Rattus norvegicus. Animal Behaviour, 76, 801-809. http://dx.doi.org/10.1016/j.anbehav.2008.02.014

Douglas, C., Bateson, M., Walsh, C., Bédué, A., \& Edwards, S. A. (2012). Environmental enrichment induces optimistic cognitive biases in pigs. Applied Animal Behaviour Science, 139, 65-73. http://dx.doi.org/10.1016/j.applanim.2012.02.018

Dugdale, N., \& Lowe, C. F. (2000). Testing for symmetry in the conditional discriminations of language-trained chimpanzees. Journal of the Experimental Analysis of Behavior, 73, 5-22. http://dx.doi.org/10.1901/jeab.2000.73-5

Gazes, R. P., Billas, A. R., \& Schmitt, V. (2017). Impact of stimulus format and reward value on quantity discrimination in capuchin and squirrel monkeys. Learning \& Behavior. Advance online publication. http://dx.doi.org/10.3758/s13420-017-0295-9

Gibeault, S., \& MacDonald, S. E. (2000). Spatial memory and foraging competition in captive western lowland gorillas (Gorilla gorilla gorilla). Primates, 41, 147-160. http://dx.doi.org/10.1007/BF02557796

Hallion, L. S., \& Ruscio, A. M. (2011). A meta-analysis of the effect of cognitive bias modification on anxiety and depression. Psychological Bulletin, 137, 940-958. http://dx.doi.org/10.1037/a0024355

Harding, E. J., Paul, E. S., \& Mendl, M. (2004). Animal behaviour: Cognitive bias and affective state. Nature, 427, 312. http://dx.doi.org/10.1038/427312a

Holland, P. C. (1989). Acquisition and transfer of conditional discrimination performance. Journal of Experimental Psychology: Animal Behavior Processes, 15, 154-165. http://dx.doi.org/10.1037/0097-7403.15.2.154

Hvorecny, L. M., Grudowski, J. L., Blakeslee, C. J., Simmons, T. L., Roy, P. R., ...Boal, J. G. (2007). Octopuses (Octopus bimaculoides) and cuttlefishes (Sepia pharaonis, S. officinalis) can conditionally discriminate. Animal Cognition, 10, 449-459. http://dx.doi.org/10.1007/s10071-007-0085-4 
Johnson-Ulrich, Z., Vonk, J., Humbyrd, M., Crowley, M., Wojtkowsk, E., ...Allard, S. (2016). Picture object recognition in an American black bear (Ursus Americanus). Animal Cognition, 19, 1237-1242. http://dx.doi.org/ 10.1007/s10071-016-1011-4

Keen, H. A., Nelson, O. L., Robbins, C. T., Evans, M., Shepherdson, D. J., \& Newberry, R. C. (2014). Validation of a novel cognitive bias task based on difference in quantity of reinforcement for assessing environmental enrichment. Animal Cognition, 17, 529-541. http://dx.doi.org/10.1007/s10071-013-0684-1

MacDonald, S. E. (1994). Gorillas' (Gorilla gorilla gorilla) spatial memory in a foraging task. Journal of Comparative Psychology, 108, 107-113. http://dx.doi.org/10.1037/0735-7036.108.2.107

Matheson, S. M., Asher, L., \& Bateson, M. (2008). Larger, enriched cages are associated with "optimistic" response biases in captive European starlings (Sturnus vulgaris). Applied Animal Behaviour Science, 109, 374-383. http://dx.doi.org/10.1016/j.applanim.2007.03.007

Mathews, A., Mackintosh, B., Thank, W., Clark, A., Diefenbach, G., ...Ward, S. (2000). Induced emotional interpretation bias and anxiety. Journal of Abnormal Psychology, 109, 602-615. http://dx.doi.org/10.1037//002I-843X.109.4.602

McGuire, M. C., Vonk, J., Fuller, G., \& Allard, S. (2017a). Using an ambiguous cue paradigm to assess cognitive bias in gorillas (Gorilla gorilla gorilla) during a forage manipulation. Animal Behavior and Cognition, 4, 7083. http://dx.doi.org/10.12966/abc.06.02.2017

McGuire, M. C., Vonk, J. \& Johnson-Ulrich, Z. (2017b). Ambiguous results when using the ambiguous-cue paradigm to assess learning and cognitive bias in gorillas and a black bear. Behavioral Sciences, 7, 51. http://dx.doi.org/10.3390/bs7030051

Mendl, M., Burman, O. H. P., Parker, R. M. A., \& Paul, E. S. (2009). Cognitive bias as an indicator of animal emotion and welfare: Emerging evidence and underlying mechanisms. Applied Animal Behaviour Science, 118, 161-181. http://dx.doi.org/10.1016/j.applanim.2009.02.023

Mendl, M., \& Paul, E. (2004). Consciousness, emotion and animal welfare: Insights from cognitive science. Animal Welfare, 13, S17-S25.

Paul, E. S., Harding, E. J., \& Mendl, M. (2005). Measuring emotional processes in animals: The utility of a cognitive approach. Neuroscience and Biobehavioral Reviews, 29, 469-491. http://dx.doi.org/10.1016/j.neubiorev.2005.01.002

Parron, C., Call, J., \& Fagot, J. (2008). Behavioural responses to photographs by pictorially naïve baboons (Papio anubis), gorillas (Gorilla gorilla) and chimpanzees (Pan troglodytes). Behavioural Processes, 78, 351-357. http://dx.doi.org/10.1016/j.beproc.2008.01.019

Pomerantz, O., Terkel, J., Suomi, S. J., \& Paukner, A. (2012). Stereotypic head twirls, but not pacing, are related to a "pessimistic"-like judgment bias among captive tufted capuchins (Cebus apella) Animal Cognition, 15, 689698. http://dx.doi.org/10.1007/s10071-012-0497-7

Schmitt, V., \& Fischer, J. (2011). Representational format determines numerical competence in monkeys. Nature Communications, 2. http://dx.doi.org/10.1038/ncomms1262

Thomas, R. K., \& Kerr, R. S. (1976). Conceptual conditional discrimination in Saimiri sciureus. Animal Learning \& Behavior, 4, 333-336. http://dx.doi.org/10.3758/BF03214060

Vonk, J. (2002). Can orangutans and gorillas acquire concepts for social relationships? International Journal of Comparative Psychology, 15, 257-277.

Vonk, J. (2003). Gorilla (Gorilla gorilla gorilla) and orangutan (Pongo abelii) understanding of first-and secondorder relations. Animal Cognition, 6, 77-86. http://dx.doi.org/10.1007/s10071-003-0159-x

Vonk, J. (2013). Matching based on biological categories in orangutans (Pongo abelii) and a gorilla (Gorilla gorilla gorilla). PeerJ. 1, e158. http://dx.doi.org/10.7717/peerj.158

Vonk, J., \& Hamilton, J. (2014). Orangutans (Pongo abelii) and a gorilla (Gorilla gorilla gorilla) match features in familiar and unfamiliar individuals. Animal Cognition, 17, 1089-1105. http://dx.doi.org/10.1007/s10071-014$0741-4$

Vonk, J., \& MacDonald, S. E. (2002). Natural concepts in a juvenile gorilla (Gorilla gorilla gorilla) at three levels of abstraction. Journal of the Experimental Analysis of Behavior, 78, 315-332. http://dx.doi.org/10.1901/jeab.2002.78-315

Vonk, J., Torgerson-White, L., McGuire, M., Thueme, M., Thomas, J., \& Beran, M. J. (2014). Quantity estimation and comparison in western lowland gorillas (Gorilla gorilla gorilla). Animal Cognition, 17, 755-765. http://dx.doi.org/10.1007/s10071-013-0707-y

Ward, C., \& Smuts, B. B. (2007). Quantity-based judgments in the domestic dog (Canis lupus familiaris). Animal Cognition, 10, 71-80. http://dx.doi.org/10.1007/s10071-006-0042-7 